\title{
ON JOINS WITH GROUP CONGRUENCES
}

\author{
by P. M. EDWARDS
}

(Received 29th November 1994)

\begin{abstract}
Let $\mathscr{S}$ be an arbitrary semigroup. A congruence $\gamma$ on $\mathscr{S}$ is a group congruence if $\mathscr{S} / \gamma$ is a group. The set of group congruences on $\mathscr{S}$ is non-empty since $\mathscr{S} \times \mathscr{S}$ is a group congruence. The lattice of congruences on a semigroup $\mathscr{S}$ will be denoted by $\mathscr{C}(\mathscr{S})$ and the set of group congruences on $\mathscr{S}$ will be denoted by $\mathscr{G} \mathscr{C}(\mathscr{P})$. If $\mathscr{G} \mathscr{C}(\mathscr{S})$ is a lattice then it is modular and $\gamma \vee \rho=\gamma \circ \rho=\rho \circ \gamma$ for all $\gamma, \rho \in \mathscr{G} \mathscr{B}(\mathscr{P})$. The main result is that $\gamma \vee \rho=\gamma \circ \rho \circ \gamma$ for any $\gamma \in \mathscr{G} \mathscr{C}(\mathscr{P})$ and $\rho \in \mathscr{C}(\mathscr{S})$ (whence every element of the set $\mathscr{G} \mathscr{C}(\mathscr{P}$ ) is dually right modular in $\mathscr{C}(\mathscr{S})$ ). This result has appeared, for particular classes of semigroups, many times in the literature. Also $\gamma \vee \rho=\gamma \circ \rho \circ \gamma=\rho \circ \gamma \circ \rho$ for all $\gamma, \rho \in \mathscr{G} \mathscr{C}(\mathscr{S})$ which is similar to the well known result for the join of congruences on a group. Furthermore, if $\gamma \cap \rho \in \mathscr{G} \mathscr{C}(\mathscr{S})$ then $\gamma \vee \rho=\gamma \circ \rho=\rho \circ \gamma$.
\end{abstract}

1991 Mathematics subject classification: 20 M 10

\section{Introduction and summary}

Unless stated otherwise $\mathscr{S}$ will always be an arbitrary semigroup. A congruence $\gamma$ on $\mathscr{S}$ is a group congruence if $\mathscr{S} / \gamma$ is a group. The set of group congruences on $\mathscr{S}$ is non-empty since $\mathscr{S} \times \mathscr{S}$ is a group congruence. The lattice of congruences on a semigroup $\mathscr{S}$ will be denoted by $\mathscr{C}(\mathscr{S})$ and the set of group congruences on $\mathscr{S}$ will be denoted by $\mathscr{G} \mathscr{C}(\mathscr{S})$. If $\mathscr{G} \mathscr{C}(\mathscr{S})$ is a lattice then it is modular (Corollary 4). If $\mathscr{S}$ has a minimum group congruence it will be denoted by $\sigma$. The existence of a minimum group congruence is equivalent to having a maximum homomorphic group image. If $\mathscr{P}$ has a minimum group congruence then $\mathscr{G} \mathscr{C}(\mathscr{S})$ is a complete modular lattice.

The main result [Theorem 1] is that $\gamma \vee \rho=\gamma \circ \rho \circ \gamma$ for any $\gamma \in \mathscr{G} \mathscr{C}(\mathscr{S})$ and $\rho \in \mathscr{C}(\mathscr{S})$ whence every element of the set $\mathscr{G} \mathscr{C}(\mathscr{S})$ is dually right modular in $\mathscr{C}(\mathscr{S})$. Also $\gamma \vee \rho=\gamma \circ \rho \circ \gamma=\rho \circ \gamma \circ \rho$ for all $\gamma, \rho \in \mathscr{G} \mathscr{C}(\mathscr{S})$ which is similar to the well known result for the join of congruences on a group. Theorem 1 is proved to apply to all semigroups and has appeared, for particular classes of semigroups, many times in the literature. That $\sigma \vee \rho=\sigma \circ \rho \circ \sigma$ for $\mathscr{S}$ inverse is [3, Theorem 3.9] and that $\gamma \vee \rho=\gamma \circ \rho \circ \gamma$ for all $\gamma=\mathscr{G} \mathscr{C}(\mathscr{S})$ for $\mathscr{S}$ regular is [6, Theorem 6]. Other usage appears in [5, Section 3], [6, Section 2], [7, Section 6], [8, Lemma III.5.4] and the regular case was generalised in [2, Theorem 5].

Results concerning joins of congruences and group congruences for specific classes of semigroups can be found in $[3,7,8]$ for inverse semigroups, [5, 6] for regular semigroups, $[5,6]$ for orthodox semigroups, and [2] for eventually regular semigroups. 


\section{Congruences on groups}

Obviously when $\mathscr{S}$ is a group, $G$ say, $\mathscr{G} \mathscr{C}(G)=\mathscr{C}(G)$. It is well known that for $G=G_{e}$ there is a one to one correspondence between the normal subgroups of $G$ and the congruences on $G$. Explicitly, if $N$ is a normal subgroup of $G$ then $\rho_{N}=\left\{(a, b) \in G \times G: a b^{-1} \in N\right\}$ is a congruence on $G$ and $N=e \rho_{N}$. Conversely if $\rho$ is a congruence on $G$ then $N=e \rho$ is a normal subgroup of $G$. Furthermore if $M$ and $N$ are normal subgroups of $G$ then $\rho_{N} \cap \rho_{M}=\rho_{N \cap M}$ and $\rho_{N} \circ \rho_{M}=\rho_{N M}$. Thus $\rho_{N} \circ \rho_{M}=\rho_{N M}=\rho_{M N}=\rho_{M} \circ \rho_{N}$ and so $\mathscr{G} \mathscr{C}(G)$ is a modular lattice and $\gamma \vee \rho=\gamma \circ \rho=\rho \circ \gamma$ for all $\gamma, \rho \in \mathscr{G} \mathscr{C}(G)$.

In the next section joins of congruences on an arbitrary semigroup will be considered. Results will be given for the case when one (or both) of the congruences is a group congruence.

\section{Joins with group congruences}

In general the join of two congruences on a semigroup $\mathscr{S}$ may be quite complicated. In fact for $\rho, \beta \in \mathscr{C}(\mathscr{S}), \rho \vee \beta$ is the transitive closure of $\rho \cup \beta$ and so $\rho \vee \beta=(\rho \circ \beta)^{\infty}=\bigcup_{n=1}^{\infty}(\rho \circ \beta)^{n}$. Example 1 below demonstrates a semigroup $\mathscr{S}$ and $\rho, \beta \in \mathscr{C}(\mathscr{S})$ for which this union must be infinite. Theorem 1 below shows that we can do much better if one of the congruences is a group congruence. The previous section mentioned how the first term of the union suffices when $\mathscr{S}$ is a group.

Theorem 1. Let $\gamma$ be a group congruence on an arbitrary semigroup $\mathscr{S}$ and let $\rho$ be a congruence on $\mathscr{S}$. Then $\gamma \vee \rho=\gamma \circ \rho \circ \gamma$.

Proof. It suffices to show that $\gamma \circ \rho \circ \gamma$ is transitive. Let $i \gamma$ denote the identity element of the group $\mathscr{S} / \gamma$. Take any $(x, y),(y, z) \in \gamma \circ \rho \circ \gamma$. It will be shown that $(x, z) \in \gamma \circ \rho \circ \gamma$. Then there exist $a, b, c, d \in \mathscr{S}$ such that $(x, a) \in \gamma,(a, b) \in \rho,(b, y) \in \gamma$, $(y, c) \in \gamma,(c, d) \in \rho$ and $(d, z) \in \gamma$. Clearly $(b, c) \in \gamma$ and so there exists $s \gamma$ in $\mathscr{S} / \gamma$ such that $s \gamma b \gamma=i \gamma$ and $c \gamma s \gamma=i \gamma$. Since $(a, b) \in \rho$ and $(c, d) \in \rho,(c s a, c s b) \in \rho$ and $(c s b, d s b) \in \rho \quad$ whence $\quad(c s a, d s b) \in \rho$. Therefore $\quad x \gamma a \gamma c s a \rho d s b \gamma d \gamma z$ whence $(x, z) \in \gamma \circ \rho \circ \gamma$.

In [5] a modularity relation $M$ was defined on a lattice $L$ by $a M b$ if $(x \vee a) \wedge b=x \vee(a \wedge b)$ for all $x \leq b$; with its dual denoted $M^{*}$. An element $d$ is right [left] modular if $a M d$ [dMa] for all $a \in L$. If $L$ is right and left modular then it is modular. Proposition 2.3 of [5] states that if $\alpha, \beta, \xi \in \mathscr{C}(\mathscr{S})$ and $\alpha \vee \beta=\alpha \circ \beta \circ \alpha$ then $\beta M^{*} \alpha$ whence (i) if $\alpha \vee \xi=\alpha \circ \xi \circ \alpha$ for all $\alpha \in \mathscr{C}(\mathscr{P})$ then $\xi$ is (dually) left modular and (ii) if $\alpha \vee \xi=\xi \circ \alpha \circ \xi$ for all $\alpha \in \mathscr{C}(\mathscr{S})$ then $\xi$ is dually right modular.

Corollary 2. For any semigroup $\mathscr{S}$, every group congruence on $\mathscr{S}$ is a dually right modular element of the lattice of congruences on $\mathscr{S}$. 
Corollary 3. Let $\gamma$ and $\rho$ be a group congruences on an arbitrary semigroup $\mathscr{S}$. Then $\gamma \vee \rho=\gamma \circ \rho \circ \gamma=\rho \circ \gamma \circ \rho$. Furthermore if $\gamma \cap \rho \in \mathscr{G} \mathscr{C}(\mathscr{S})$ then $\gamma \vee \rho=\gamma \circ \rho=\rho \circ \gamma$.

Proof. The first assertion follows from Theorem 1. Suppose $\beta=\gamma \cap \rho \in \mathscr{G} \mathscr{C}(\mathscr{P})$ and put $L=[\beta, \mathscr{S} \times \mathscr{S}]$. Then $L \cong \mathscr{C}(\mathscr{S} / \beta)$ which is a modular lattice of commuting congruences since $\mathscr{S} / \beta$ is a group. Thus $\gamma$ and $\rho$ commute.

Corollary 4. If $\mathscr{G} \mathscr{C}(\mathscr{S})$ is a sublattice of $\mathscr{C}(\mathscr{S})$ then $\mathscr{G} \mathscr{C}(\mathscr{S})$ is a modular lattice and $\gamma \vee \rho=\gamma \circ \rho=\rho \circ \gamma$ for all $\gamma, \rho \in \mathscr{G} \mathscr{C}(\mathscr{S})$.

The kernel of $\rho \in \mathscr{C}(\mathscr{S})$ is defined by, $\operatorname{ker}(\rho)=\{a \in \mathscr{S}: a \rho \in E(\mathscr{S} / \rho)\}$. It is well known that if two group congruences have the same kernel then they are equal. For a subset $H$ of $\mathscr{S}$, define $H \omega=\{x \in \mathscr{S}: h x \in H$ for some $h \in H\}$, define $\omega H$ dually and put $H^{\prime}=\omega H \cup H \omega$. If $H$ is a subsemigroup then $H \subseteq H \omega$ and $H \subseteq \omega H$. The subset $H$ is called closed if $H=H^{\prime}$. It is straightforward to show that $H=\operatorname{ker}(\gamma)$ is always a closed subsemigroup for $\gamma \in \mathscr{G} \mathscr{C}(\mathscr{S})$.

After proving the following preliminary result some applications of Theorem 1 will be given.

Theorem 5. Let $\gamma$ be a group congruence on an arbitrary semigroup with $H=\operatorname{ker}(\gamma)$. Then the following are equivalent:

(1) $a$ y $b$,

(2) $x a=b y$ for some $x, y \in H$,

(3) $a x=y b$ for some $x, y \in H$,

(4) $H a H \cap H b H \neq \emptyset$.

Proof. Suppose $a \gamma b$. Let $z$ be the group inverse of $a \gamma=b \gamma$ in $\mathscr{S} / \gamma$ and put $x=b z$, $y=z a, s=z b$ and $t=a z$. Then $x a=b z a=b y$ and $a s=a z b=t b$ and $x, y, s, t \in \operatorname{ker}(\gamma)$ whence (1) implies (2) and (3). It also follows that for any $h \in H, h b z a h=(h b z) a h=$ $h b(z a h) \in H a H \cap H b H$ so (1) implies (4). That (2), (3) and (4) each imply (1) is trivial, whence the four statements are equivalent.

Theorem 6. For any congruence $\rho$ and any group congruence $\gamma$ on an arbitrary semigroup, $a(\gamma \vee \rho) b$ if and only if $x a \rho$ by for some $x, y \in \operatorname{ker}(\gamma)$.

Proof. The following proof is a slight modification of the proof of Theorem 7 of LaTorre [6]. Put $H=\operatorname{ker}(\gamma)$. Suppose $(a, b) \in \gamma \vee \rho$. By Theorem 1 there exist $c, d \in \mathscr{S}$ such that $a \gamma c, c \rho d$ and $d \gamma b$. Since $a \gamma c$, by Theorem 5 there exist $h, k \in H$ such that $h a=c k$ and similarly there exist $p, q \in H$ such that $p d=b q$. Put $x=p h$ and $y=q k$ so $x, y \in H$. Then $x a=p h a=p(h a)=p(c k) \rho p(d k)=(p d) k=(b q) k=b y$ so $x a \rho b y$ with $x, y \in H$. Conversely, if $x a \rho b y$ with $x, y \in H$ then since $x a \gamma a$ and $b y \gamma a, a \gamma x a \rho b y \gamma b$ so $(a, b) \in \gamma \vee \rho$. 
Theorem 7. For any congruence $\rho$ and any group congruence $\gamma$ on an arbitrary semigroup, $\operatorname{ker}(\gamma \vee \rho)=((\operatorname{ker}(\gamma)) \rho) \omega$.

Proof. Take $x \in \operatorname{ker}(\gamma \vee \rho)$. For any $e \in E,(x, e) \in \gamma \vee \rho$ so from Theorem 6 there exist $p, q \in \operatorname{ker}(\gamma)$ such that $x p \rho q e$. Now $q e \in \operatorname{ker}(\gamma)$ so $x p \in(\operatorname{ker}(\gamma)) \rho$. Since $p \in \operatorname{ker}(\gamma) \subseteq(\operatorname{ker}(\gamma)) \rho$, we have that $x \in((\operatorname{ker}(\gamma)) \rho) \omega$. Conversely, if $x \in((\operatorname{ker}(\gamma)) \rho) \omega$, then $h x \in(\operatorname{ker}(\gamma)) \rho$ for some $h \in(\operatorname{ker}(\gamma)) \rho$ so $(h x, y) \in \rho$ for some $y \in \operatorname{ker}(\gamma)$ and $(h, z) \in \rho$ for some $z \in \operatorname{ker}(\gamma)$. Since $(y, e) \in \gamma$, it follows that $(h x, e) \in \gamma \vee \rho$ so $h x \in \operatorname{ker}(\gamma \vee \rho)$. Similarly, $h \in \operatorname{ker}(\gamma \vee \rho)$ whence $x \in \operatorname{ker}(\gamma \vee \rho)$ since $\operatorname{ker}(\gamma \vee \rho)$ is closed. Therefore $\operatorname{ker}(\gamma \vee \rho)=((\operatorname{ker}(\gamma)) \rho) \omega$.

Theorems 1, 6 and 7 above generalise the corresponding results for regular semigroups given in [6].

Example 1. Let $\mathscr{S}=\mathbb{Z}^{+}$with left zero multiplication and let $\rho$ and $\beta$ be congruences on $\mathscr{S}$ given respectively by the partitions $\{\{1\},\{2,3\},\{4,5\},\{6,7\}, \ldots\}$ and $\{\{1,2\},\{3,4\},\{5,6\}, \ldots\}$. Then $\rho \vee \beta=\mathscr{S} \times \mathscr{S}$ and clearly $\rho \vee \beta \neq \bigcup_{n=1}^{m}(\rho \circ \beta)^{n}$ for any finite $m$.

Example 2. A congruence $\gamma$ on $\mathscr{S}$ is a cancellative congruence if $\mathscr{S} / \gamma$ is a cancellative semigroup. Any semigroup has a minimum cancellative congruence, namely the intersection of all the cancellative congruences. Denote the set of idempotent elements in $\mathscr{S}$ by $E=E(\mathscr{S})$. A semigroup $\mathscr{S}$ is $E$-inversive if for all $x \in \mathscr{S}$, there exists $y \in \mathscr{S}$ such that $x y \in E$. A semigroup is eventually regular [group-bound] if every element has some power that is regular [in a subgroup]. The class of $E$-inversive semigroups includes eventually regular semigroups, regular semigroups, group-bound semigroups, finite semigroups and semigroups with a zero element. If $\mathscr{P}$ is an $E$-inversive semigroup then the group congruences coincide with the cancellative congruences, whence $\mathscr{S}$ has a minimum group congruence $\sigma$ equal to the intersection of all cancellative congruences on $\mathscr{S},[1]$. Let $\mathscr{S}$ be any semigroup that possesses a minimum group congruence $\sigma$. Then $\mathscr{G} \mathscr{C}(\mathscr{S})=[\sigma, \mathscr{S} \times \mathscr{S}]$ is a complete modular lattice. The mapping $\phi: \mathscr{C}(\mathscr{P}) \rightarrow \mathscr{G} \mathscr{C}(\mathscr{P})$ defined by $\phi(\rho)=\sigma \vee \rho$ is a surjection of $\mathscr{C}(\mathscr{S})$ onto $\mathscr{G} \mathscr{C}(\mathscr{S})$ and the elements of $\mathscr{G} \mathscr{C}(\mathscr{S})$ are invariant under $\phi$. If $\mathscr{S}$ is orthodox then the mapping $\phi$ is a homomorphism, [6, Theorem 11].

Example 3. Let $\mathscr{S}=\langle a\rangle$ be an infinite monogenic semigroup. Then the relation $\rho_{n}=\left\{\left(a^{p}, a^{q}\right): p \equiv q(\bmod n)\right\}$ is a group congruence on $\mathscr{S}$ and every group congruence on $\mathscr{S}$ is of this form [4, p. 185, Exercise 26]. If $\rho_{n}$ and $\rho_{m}$ are two group congruences on $\mathscr{S}$ then $\rho_{n} \cap \rho_{m}=\rho_{k}$ where $k$ is the lowest common multiple of $n$ and $m$. It follows that the intersection of any finite set of group congruences on $\mathscr{S}$ is a group congruence and that $\mathscr{S}$ does not possess a minimum group congruence. In fact the intersection of all group congruences on $\mathscr{S}$ equals $1_{\mathscr{S}}$ which is of course not a group congruence. Therefore $\mathscr{G} \mathscr{C}(\mathscr{P})$ is a lattice but is not a complete lattice. It follows from Corollary 4 that $\mathscr{G} \mathscr{C}(\mathscr{S})$ is a modular lattice. 
Example 4. This example was suggested by T. E. Hall. Put $A=\langle a\rangle$ and $B=\langle b\rangle$ both infinite cyclic groups and in $A \times B$ let $\mathscr{S}$ be the subsemigroup generated by $(a, b),(a, b$ inverse) and ( $a$ inverse, $b)$. Note that if $\left(a^{p}, b^{q}\right) \in \mathscr{S}$ then $p+q$ is even and $p+q \geq 0$. In particular, the semigroup $\mathscr{S}$ is not a group since $(a, b)$ does not have an inverse in $\mathscr{S}$. Let $\rho_{A}$ and $\rho_{B}$ be the kernels of the projections of $\mathscr{S}$ onto $A$ and $B$ respectively. Then $\rho_{A}$ and $\rho_{B}$ are group congruences on $\mathscr{S}$ but their intersection is trivial and so is not a group congruence. Since $\left(a^{2}, b^{-2}\right) \rho_{A}\left(a^{2}, b^{0}\right) \rho_{B}\left(a^{0}, b^{0}\right)$ we have that $\left(a^{2}, b^{-2}\right)$ is $\rho_{A} \circ \rho_{B}$ related to $\left(a^{0}, b^{0}\right)$. Because $\left(a^{0}, b^{-2}\right) \notin \mathscr{S},\left(a^{2}, b^{-2}\right)$ is not $\rho_{B} \circ \rho_{A}$ related to $\left(a^{0}, b^{0}\right)$. Thus $\rho_{A} \circ \rho_{B} \neq \rho_{B} \circ \rho_{A}$ [cf. Corollary 3].

\section{REFERENCES}

1. A. H. Clifford and G. B. Preston, The algebraic theory of semigroups, Volume I (Mathematical Surveys, 7, American Mathematical Society, Providence, Rhode Island, 1961).

2. S. Hanumantha RaO and P. Lakshmi, Group congruences on eventually regular semigroups, J. Austral. Math. Soc. Ser. A 45 (1988), 320-325.

3. J. M. HowIE, The maximum idempotent-separating congruence on an inverse semigroup, Proc. Edinburgh Math. Soc. 14 (1964), 71-79.

4. J. M. Howle, An introduction to semigroup theory (London Mathematical Society Monographs, 7, Academic Press, London, New York, San Francisco, 1976).

5. P. R. Jones, Joins and meets of congruences on a regular semigroup, Semigroup Forum 30 (1984), 1-16.

6. D. R. LATorre, Group congruences on regular semigroups, Semigroup Forum 24 (1982), 327-340.

7. Mario Petrich, Congruences on inverse semigroups, J. Algebra 55 (1978), 231-256.

8. Mario Petrich, Inverse semigroups (John Wiley, New York, 1984).

\section{ECONOMETRICS DEPARTMENT}

MONASH UNIVERSITY

Clayton

VICTORIA 3168

Australia 